\title{
Characterization of Viscoelastic Soft Tissue Properties from In vivo Animal Experiments and Inverse FE Parameter Estimation
}

\author{
Jung $\mathrm{Kim}^{1}$ and Mandayam A. Srinivasan ${ }^{2}$ \\ ${ }^{1}$ Dept. of Mechanical Engineering, \\ Korea Advanced Institute of Science and Technology, Korea \\ 2 The Touch Lab, Dept of Mechanical Engineering, \\ Massachusetts Institute of Technology, USA \\ jungkim@kaist.ac.kr, srini@mit.edu
}

\begin{abstract}
Soft tissue characterization and modeling based on living tissues has been investigated in order to provide a more realistic behavior in a virtual reality based surgical simulation. In this paper, we characterize the nonlinear viscoelastic properties of intra-abdominal organs using the data from in vivo animal experiments and inverse FE parameter estimation algorithm. In the assumptions of quasi-linear-viscoelastic theory, we estimated the viscoelastic and hyperelastic material parameters to provide a physically based simulation of tissue deformations. To calibrate the parameters to the experimental results, we developed a three dimensional FE model to simulate the forces at the indenter and an optimization program that updates new parameters and runs the simulation iteratively. We can successfully reduce the time and computation resources by decoupling the viscoelastic part and nonlinear elastic part in a tissue model. The comparison between simulation and experimental behavior of pig intra abdominal soft tissue are presented to provide a validness of the tissue model using our approach.
\end{abstract}

\section{Introduction}

Soft tissue characterization and modeling has been investigated in order to understand mechanisms of traumatic injury. For example, Farshad et al. [1] characterized the material parameters of pig kidney based on uniaxial tension tests on ex vivo samples. They were mainly interested in a tissue behavior under high speed or impact loading conditions, which occur in accidents, so their measurements were mainly ex vivo measurements. Recent developments in medical instruments have motivated the tissue characterization under the lower speed loading conditions and using the data from in vivo measurements which are expected to provide the properties of living tissues. Davies et al. [2] developed a two dimensional mathematical model for pig spleen tissue from uniform compression tests using a large-area indenter. They solved the nonlinear constrained boundary value problems numerically with an exponential stress-strain law and a finite element model (FEM). By varying the model size, they showed that the forces measured at the indenter were insensitive to the size of the model. Miller [3] developed a three-dimensional, hyperelastic, viscoelastic 
constitutive model for abdominal organ tissues. The model was developed from a strain-energy function with time dependent constants.

Although in vivo experiments can provide the data in a living state and they are essential to develop soft tissue models for surgical simulation or rehearsal, the characterization of soft tissue properties has not been successful. Because the target organs have non-uniform cross-sectional area and non-uniform strain across any given cross section. An analytic solution based on the boundary value problem was not a good candidate, given the complexity of the material behavior, the organ geometry, and the three-dimensional deformation imposed on the surface. To circumvent these difficulties, inverse finite element estimation has been investigated recently. This method estimates unknown material parameters for a selected material law by minimizing the least-squares difference between predictions of a finite element model and experimental responses. In this paper, we estimated viscoelastic material properties of soft tissue by using the in vivo animal experimental data and a FE tool to provide a model for medical simulation or off-line analysis.

\section{In vivo Animal Experiments}

We measured pig's liver and kidney under open surgical conditions to measure mechanical properties in vivo at the Harvard Center for Minimally Invasive Surgery in collaboration with surgeons from the Massachusetts General Hospital (MGH). A total of 10 pigs were used in these experiments. The pigs were first put under general anesthesia and placed on the surgical table. A midline incision was then made at its abdominal region and dissection carried out on the anatomical structures to expose the organs. The indentation stimuli were delivered using the haptic interface device, Phantom Premium-T 1.5 (SensAble Technologies, www.sensable.com) that was programmed to perform as a mechanical stimulator. Reaction forces were measured using a six-axis force transducer, Nano 17 (ATI Industrial Automation) that was attached to the tip of the Phantom. The transducer has a force resolution of $0.781 \mathrm{mN}$ along each of the three orthogonal axes when connected to a 16-bit A/D converter. The indenter was a $2 \mathrm{~mm}$ diameter flat-tipped cylindrical probe that was fixed to the tip of the Phantom with the force transducer mounted in-between to accurately sense the reaction forces.

\section{Estimation of Soft Tissue Properties}

We used the quasi-linear viscoelasticity (QLV) framework proposed by Fung [5]. This approach assumes material behavior can be decoupled into two effects: a timeindependent elastic response, and a linear viscoelastic stress relaxation response. These models can be determined separately from the experiments. The stresses in the tissues, which may be linear or nonlinear, are linearly superposed with respect to time. by,

The three-dimensional constitutive relationship in the framework of QLV is given

$$
S(t)=G(t) S^{e}(0)+\int_{0}^{t} G(t-\tau) \frac{\partial S^{e}(E(\lambda))}{\partial \tau} d \tau
$$


where $\mathrm{S}(\mathrm{t})$ is the second Piola-Kirchhoff stress tensor and $\mathrm{G}(\mathrm{t})$ is called the reduced relaxation function. $S^{e}(E(\lambda))$ is called the pure elastic response of the material and can be nonlinear or linear. The reduced relaxation function $G(t)$ is a scalar function of time and can be often expressed by the Prony series,

$$
\begin{aligned}
& G(t)=G_{0}\left(1-\sum_{i=1}^{N} \bar{g}_{i}^{P}\left(1-\exp \left(-\frac{t}{\tau_{\mathrm{i}}}\right)\right)\right) \\
& G_{0}=G(0)
\end{aligned}
$$

where $\bar{g}_{i}^{P}$ are the Prony series parameters.

For the nonlinear elastic response, we have used an incompressible hyperelastic material representation, which is commonly used for elastomer modeling. The material properties of a hyperelastic material can be determined by a strain energy function $\mathrm{W}$. Ideally, $\mathrm{W}$ is defined with only as many parameters as are required in order to make a FE model. There are many specific material models that could be used, depending on how to approximate the strain energy function. The strain energy function of the three-dimensional incompressible Mooney-Rivlin model is given by

$$
W=C_{10}\left(I_{1}-3\right)+C_{01}\left(I_{2}-3\right)
$$

where $C_{10}, C_{01}$ are material parameters (having unit of stress) and $I_{1}, I_{2}$ are principal invariants. Since the analytical solution considering the above material law and experimental conditions is very difficult, the Finite Element Method (FEM) has been widely used in simulation. Through modeling of indentation experiments with the QLV approach, the final outcome of the FE simulation can be simply expressed as,

$$
\begin{aligned}
F s & =F E M M_{-} \operatorname{SIMULATION}\left(p_{i}\right) \\
p_{i} & =\left[\tau_{i}, \bar{g}_{i}^{P}, C_{i j}\right]
\end{aligned}
$$

where $F_{s}$ is the simulated force and $p_{i}$ is the material parameter containing the viscoelasticity and nonlinear elasticity. For example, the number of the estimated parameters for the Mooney-Rivlin model is two. The goal of the characterization is to determine these parameters for a proposed material law by minimizing the errors between the simulated and the associated experimental measurements. This process is also called the inverse calculation because it is the opposite of an ordinary simulation (that is, solving for forces or displacements given material parameters and boundary conditions).

Instead of estimating all required parameters in one step, we separated the characterization process into two stages. In the first step the viscoelastic parameters were determined from the normalized force responses against ramp-and-hold indentation from the experiments. With the viscoelastic parameters estimated in the first stage, the inverse FEM parameter estimation method was used to determine the remaining elastic parameters.. This separation of parameters is also similar to the work by Kauer et al.[6]. However, they fixed the time constants of the stress relaxation 


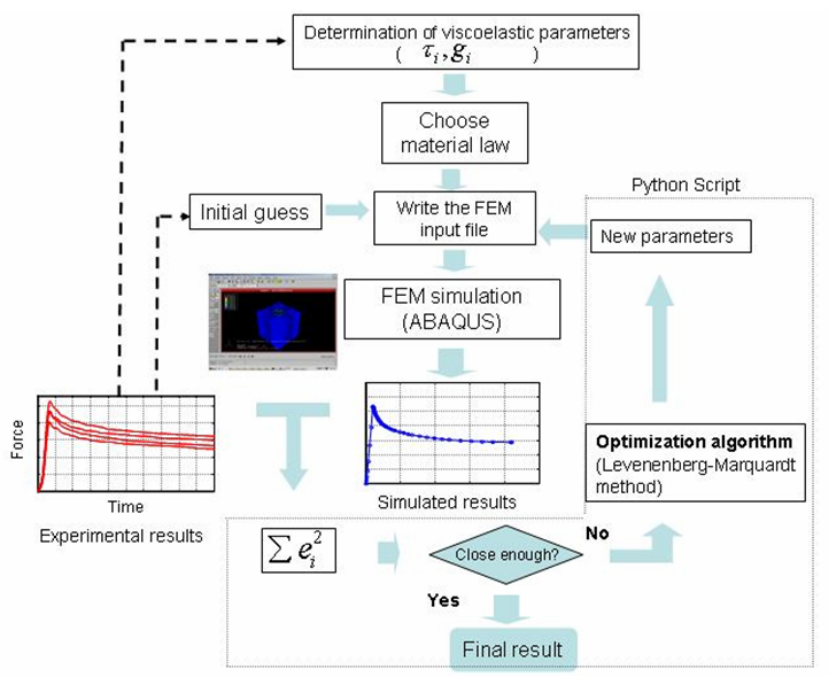

Fig. 1. Flow chart for the developed parameter estimation algorithm

$(0.1 \mathrm{~s}, 1 \mathrm{~s}, 10 \mathrm{~s}$, and $100 \mathrm{~s})$, and fit their magnitudes, then determined the rest of the parameters using an inverse FEM calculation. In our work, the parameters in the viscoelastic model were estimated directly from the normalized force-displacement data in the experiments. The overall procedures of the characterization are illustrated in Fig. 1.

\subsection{Determination of Viscoelastic Parameters}

In this section we develop a three-dimensional linear viscoelastic model of the soft tissue based on the force-displacement experiment data. The simplest lumped parameter model that can capture the viscoelastic behavior of a solid is the three parameter linear solid model whose transfer function may be written as

$$
\frac{F(s)}{\delta(s)}=F_{s s} \prod_{i=1}^{n}\left(\frac{1+\alpha_{\mathrm{i}} \tau_{\mathrm{i}} \mathrm{s}}{1+\tau_{\mathrm{i}} \mathrm{s}}\right)
$$

where $F(s)$ and $\delta(s)$ are the Laplace transformed force and displacement variables, $F_{s s}$ is the steady state value of the force response. $\tau$ is the relaxation time constant and $\alpha$ is the ratio of the initial response of the system to a step in displacement to the steady state value $(\alpha>1)$. Incidentally, this model has the similar to the Kelvin model. The point indenter is a good choice for model parameter estimation since it is the closest approximation of a punch on an elastic half space. From linear elastostatics we know that the total force, $\mathrm{P}$, required to indent a frictionless circular cylindrical punch, of radius ' $a$ ', into an isotropic elastic halfspace, by a distance $\mathrm{D}$ is given by 


$$
P=\frac{8 a G(G+3 K)}{4 G+3 K} D
$$

where $\mathrm{G}$ is the rigidity modulus, $\mathrm{a}$ is the diameter of the indenter and $\mathrm{K}$ is the bulk modulus.

From the correspondence principle and the incompressibility assumption, the above equation can be simplified,

$$
P(s)=8 \text { as } \bar{G}(s) D(s)
$$

From the above equations, the step response rigidity modulus may be written as,

$$
\bar{G}(s)=\frac{F_{s s}}{8 a s} \prod_{i=1}^{2}\left(\frac{1+\alpha_{\mathrm{i}} \tau_{\mathrm{i}} \mathrm{s}}{1+\tau_{\mathrm{i}} \mathrm{s}}\right)
$$

Using the above model and the experimental data, we can find $g_{i} \tau_{i}$ by using lsqnonlin.m, which is a built-in function for the nonlinear curve fitting in MATLAB. This approach allows the rigidity modulus to be expressed as a Prony series expansion in the time domain. Table 1 lists the computed Prony series parameters of the selected experiments.

\subsection{Estimation of Nonlinear Elastic Parameters}

In our application, the compared quantities are the simulated forces from the FEM simulation and the associated experimental forces at the indenter. Therefore, we can minimize a nonlinear sum of squares given by

$$
\begin{aligned}
\vec{E} & =\sum_{i}^{m}\left(\vec{F}_{s}\left(t_{i}\right)-\vec{F}_{e}\left(t_{i}\right)\right) \cdot\left(\vec{F}_{s}\left(t_{i}\right)-\vec{F}_{e}\left(t_{i}\right)\right) \\
t_{i} & =\left(t_{1}, t_{2} \ldots t_{m}\right)
\end{aligned}
$$

where $\mathrm{Fe}, \mathrm{Fs}, t_{i}, \mathrm{~m}$ are measured forces, simulated forces, time and the total number of data points, respectively. Among several optimization algorithms that could be used, we adopt the nonlinear least square optimization known as the MarquardtLevenberg algorithm.

Although the Levenberg-Marqudt has been used successfully in finite strain applications [7], the entire process is computationally expensive. The FEM simulation, in which a few hours per run, is repeated as many times as necessary until

Table 1. Prony series parameters from the normalized force data

\begin{tabular}{|c|c|c||c|c||c|c|}
\hline Organ & Depth $(\mathbf{m m})$ & $\tau_{1}(\mathrm{sec})$ & $\tau_{2}(\mathrm{sec})$ & $g_{1}$ & $g_{2}$ & $\sum \varepsilon^{2}$ \\
\hline Liver & 6 & 1.537 & 6.090 & 0.2866 & 0.2022 & 0.0754 \\
\hline Kidney & 5 & 0.741 & 6.171 & 0.2054 & 0.2725 & 0.0653 \\
\hline Kidney & 4 & 0.747 & 6.021 & 0.2305 & 0.2105 & 0.0867 \\
\hline
\end{tabular}


the simulated results match the experimental results. The Jacobian vector is calculated at each iteration but this requires perturbing one parameter, running the entire FEM simulation, and measuring the effect of the perturbation. To construct the vector, this must be repeated for each parameter. Thus, for five free parameters, the FE model must be solved six times per iteration (the sixth solution is the reference to which the perturbations are compared). The entire characterization process takes several iterations to converge so the computational time is very large and hence it is better to reduce the number of parameters as much as possible as in our approach. It was this computational expense that led us to identify the viscoelastic parameters before undertaking the inverse FEM simulation. This allowed us to iterate using only two (Mooney-Rivlin) free parameters. Fig. 2 shows the developed model in ABAQUS. In our experiments, the deformation field appeared to be insensitive to the organ geometry. This made sense, since our indentations were small (millimeters) compared to the organs (centimeters). Accordingly, we simplified our analysis by modeling a subdomain of the organ. The size and the mesh density of the model are carefully adjusted to ensure a well-conditioned solution. Nonlinearities from both the material model and from large geometric deformation were allowed.

The contact between the indenter and tissues was modeled with a contact mechanics module in ABAQUS and the non-uniform element density over the model was used to improve accuracy of the contact region. We implemented the LevenbergMarquardt algorithm with the Python language, which is a way to control the inputs and outputs of ABAQUS.

With the approximate initial values from the ABAQUS evaluation function, we used our Python code to iterate the FE model and update the parameters automatically. The parameters reached convergence with four or five iterations. Table 2 presents the initial parameters and converged parameters for both hyperelastic material models. With a good guess from a priori knowledge of the parameters, the parameters converged with three or four iterations in most of cases. Fig. 3 shows the predicted forces from the FE simulation with the estimated parameters and experimental forces for the pig liver and kidney. The force responses of the hyperelastic model in ABAQUS match the experimental data well.

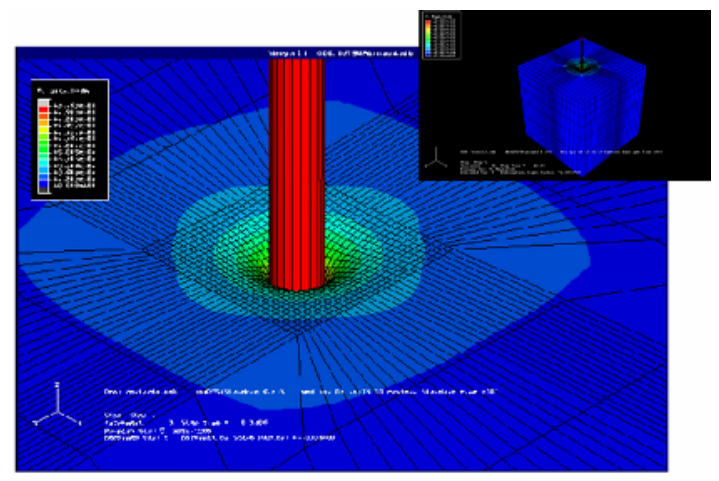

Fig. 2. FEM simulation of the experiment developed with ABAQUS. The upper right shows the shape of sub-domain region for the simulation. 
Table 2. Initial and estimated parameters in the Mooney-Rivlin model

\begin{tabular}{|l|l|l|l|l|l|l|l|}
\hline \multicolumn{2}{|c|}{ Condition } & \multicolumn{2}{|c|}{ Initial parameters } & $\begin{array}{l}\text { Iteration } \\
\text { Number }\end{array}$ & \multicolumn{2}{l|}{$\begin{array}{l}\text { Final } \\
\text { parameters }\end{array}$} & $\sum \varepsilon^{2}$ \\
\hline Organ & Indentation & $\mathrm{C} 10(\mathrm{~Pa})$ & $\mathrm{C} 01(\mathrm{~Pa})$ & & $\mathrm{C} 10(\mathrm{~Pa})$ & $\mathrm{C} 01(\mathrm{~Pa})$ & \\
\hline Liver & $6 \mathrm{~mm}$ & 83.18 & 84.76 & 3 & 322.96 & 161.47 & 0.0028 \\
\hline Kidney & $5 \mathrm{~mm}$ & 682.31 & 700.02 & 2 & 868.66 & 467.11 & 0.0031 \\
\hline
\end{tabular}
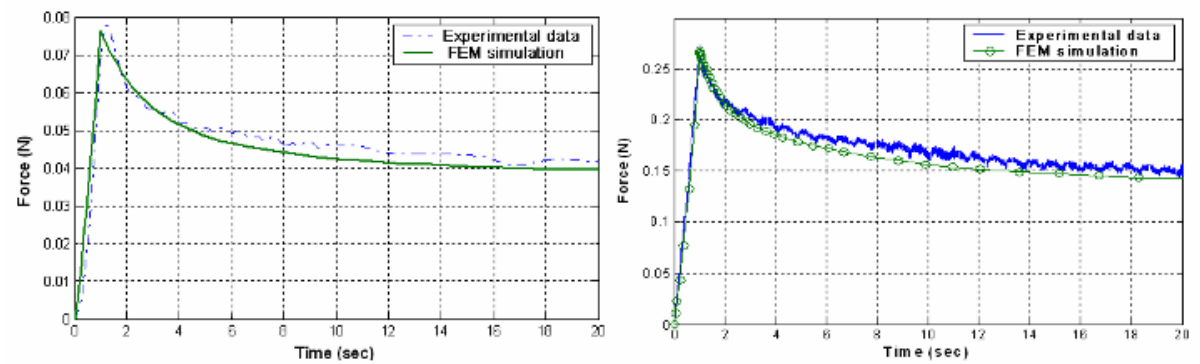

Fig. 3. Force responses of the model prediction and the experiments. (Left) liver with $5 \mathrm{~mm}$ indentation. (Right) kidney with a $6 \mathrm{~mm}$ indentation. The responses in (b) shows this noisy signal.

\section{Concluding Remarks}

In this paper, we have characterized the mechanical properties of intra-abdominal organs from the in vivo animal experiments. To calibrate the parameters to the experimental results, we developed a three dimensional FE model to simulate the forces at the indenter and an optimization program that updates new parameters and runs the simulation iteratively. Key assumptions in our approach are that the organs are incompressible, homogenous, and isotropic, and that the deformations we imposed were small compared to the size of the organ. With these limitations in mind, the material models presented in this study offer two basic uses in a VR-based medical simulation. First, they can be used directly in the simulator to compute visual deformations and interaction forces that are displayed in real time. Second, the mathematical models presented here can be used as a standard for the evaluation of new real time algorithms for computing deformation.

\section{References}

[1] M. Farshad, M. Barbezat, P. Flueler, F. Shmidlin, P. Graber, and P. Niederer, "Material Characterization of the Pig Kidney in Relation with the Biomechanical Analysis of Rental Trauma," Journal of Biomechanics, vol. 32, pp. 411-425, 1999.

[2] P. J. Davis, F. J. Carter, D. G. Loxburgh, and A. Cuschieri, "Mathematical Model for Keyhole Surgery Simulation: spleen capsule as an elastic membrane," Journal of Theoretical Medicine, vol. 1, pp. 247-262, 1999. 
[3] K. Miller, "Constitutive Modeling of Abdominal Organs," Journal of Biomechanics, vol. 33, pp. 367-373, 1999.

[4] B. K. Tay, S. De, N. Stylopoulos, D. W. Rattner, and M. A. Srinivasan, "In vivo Force Response of Intra-abdominal Soft Tissue for the Simulation of Laparoscopic Procedures," presented at Proceedings of the MMVR Conference, 2002.

[5] Y. C. Fung, Biomechanics: Mechanical Properties of Living Tissues. New York: SpringerVerlag, 1993.

[6] M. Kauer, V. Vuskovic, J. Dual, G. Szekely, and M. Bajka, "Inverse Finite Element Characterization of Soft Tissue," presented at Medical Image Computing and ComputerAssisted Intervention - MICCAI 2001, 2001.

[7] P. Seshaiyer and J. D. Humphrey, "A Sub-Domain Inverse Finite Element Characterization of Hyperelastic Membranes Including Soft Tissues," Journal of Biomechanical Engineering, Transaction of the ASME, vol. 125, pp. 363-371, 2003. 